\title{
«Das Bundesgericht erhebt die alternierende Obhut zur Regel»
}

Besprechung der Urteile des Bundesgerichts 5A_367/2020 vom 19. Oktober 2020 und 5A_629/2019 vom 13. November 2020

Mit Urteilen von 19. Oktober und 13. November 2020 verdeutlicht das Bundesgericht seine bisherige Rechtsprechung zu strittigen Betreuungsfragen: Es nimmt die alternierende Obhut zum Ausgangspunkt der Entscheidfindung. Zudem priorisiert es gleiche Betreuungsanteile gegenüber ungleichen. Die alleinige Obhut ist anzuordnen, wenn im Einzelfall konkrete Gründe gegen hälftige Betreuungsanteile sprechen. Abweichungen von dieser Rechtsprechung können gegen das Willkürverbot verstossen. Das Bundesgericht gibt der alternierenden Obhut damit tendenziell den Vorzug in Betreuungsfragen. Der Beitrag analysiert die Bedeutung der Urteile für die Praxis und klärt offene Fragen zur «alternierenden Obhut als Regelfall» sowie zum Willen des Gesetzgebers.

I. Übersicht

II. Grundsätze zur Ermittlung der Betreuungsform

III. Urteil 5A_629/2019 vom 13. November 2020

IV. Urteil 5A_367/2020 vom 19. Oktober 2020

V. Bedeutung für die Praxis

VI. Berichtigung zur «Regelfall-Diskussion»

VII. Der Wille des Gesetzgebers

VIII. Würdigung

IX. Fazit

Zitiervorschlag:

MARTIN WIDRIG, «Das Bundesgericht erhebt die alternierende Obhut zur Regel», sui generis 2021, S. 197

Martin Widrig, MLaw, Lektor an der Universität Freiburg (martin.widrig@unifr.ch).

URL: sui-generis.ch/183

DOI: https://doi.org/10.21257/sg.183

Dieses Werk ist lizenziert unter einer Creative Commons Namensnennung - Weitergabe unter gleichen Bedingungen 4.0 International Lizenz. 


\section{I. Übersicht}

1 «(D)as BGer erhebt die altern. Obhut mit gleichen Anteilen faktisch zum Regelfall» twitterte Prof. Fankhauser zum Urteil des Bundesgerichts 5A_629/2019 vom 13. November 2020, was zum Titel des vorliegenden Beitrags inspirierte. ${ }^{1}$ In diesem Entscheid hiess unser höchstes Gericht die Beschwerde eines Vaters gut, der anstelle eines ausgeweiteten Besuchsrechts eine alternierende Obhut mit hälftiger Betreuungsaufteilung wünschte. Da die Vorinstanz die alternierende Obhut ablehnte, ohne eine Beeinträchtigung des Kindeswohls festzustellen und rechtserhebliche Umstände ausser Acht liess, wies das Bundesgericht die Rechtssache zur Ergänzung des Sachverhalts und Neubeurteilung an sie zurück.

2 Auch mit seinem Urteil5A_367/2020 vom 19. Oktober 2020 bestätigte das Bundesgericht deutlich seine Rechtsprechung, wonach eine alternierende Obhut im Einzelfall anzuordnen ist, wenn keine konkreten und sachlichen Gründe dagegensprechen. ${ }^{2}$ I.c. hob es den Entscheid ausschliesslich unter Willkürgesichtspunkten (!) auf und wies ihn zur Anordnung und Regelung der Modalitäten einer alternierenden Obhut an die Vorinstanz zurück.

3 Nachfolgend seien die beiden Urteile mit Fokus auf ihre Bedeutung für Betreuungsfragen analysiert. Dazu werden kurz die bundesgerichtlichen Kriterien zur Ermittlung der Betreuungsregelung wiederholt (II.) und die beiden Entscheide erläutert sowie ihre Bedeutung für die Praxis besprochen (III.-V.). Dem folgen ergänzende Hin weise zur «Regelfall-Diskussion», dem Willen des Gesetzgebers, eine kurze Würdigung sowie ein Fazit (VI.-IX.).

\section{Grundsätze zur Ermittlung der Betreuungsform}

4 Ist die Betreuungsregelung strittig, ist nach bundesgerichtlicher Rechtsprechung im Einzelfall zu entscheiden, welche Betreuungsregelung anzuordnen ist. Das massgebende Kriterium ist das Kindeswohl. ${ }^{3}$ Dieses ist wie folgt zu ermitteln:

5 Gestützt auf festgestellte Tatsachen der Gegenwart und der Vergangenheit ist in einem ersten Schritt eine Prog-

\footnotetext{
1 ROLAND FANKHAUSER, @JurBSFankhauser, Tweet vom 2. Dezem ber 2020. Vgl. auch die Interpretation des Obergerichts des Kantons Zürich (Urteil des Obergerichts des Kantons Zürich PQ200074-O/U vom 29.Januar 2021 E. 3.3).

2 BGE142 III 612 E. 4.4; BGE142 III 617 E. 3.2.4. Vgl. auch statt vieler: Urteil des Bundesgerichts 5A_888/2016 vom 20. April 2018 E.3.2.1f. und 3.3.6.

3 BGE142 III 612 E. 4.2; BGE142 III617 E.3.2.3.
}

nose darüber zu erstellen, ob eine alternierende Obhut ${ }^{4}$ mit dem Kindeswohl vereinbar ist. ${ }^{5}$ Das Bundesgericht erwähnt eine nicht abschliessende Zahl von Kriterien, auf die es bei der Erstellung dieser Prognose ankommt. Von ihm genannte Kriterien sind:

a. die Erziehungsfähigkeit der Eltern,

b. die persönliche Beziehung des Kindes zu den Eltern,

c. die Möglichkeit der Eltern, das Kind persönlich zu betreuen,

d. die Fähigkeit und Bereitschaft der Eltern, in Kinderbelangen zu kommunizieren und zu kooperieren,

e. die Bereitschaft, den Kontakt zum anderen Elternteil zu fördern,

f. das Alter des Kindes,

g. die geografische Situation; namentlich die Distanz zwischen den Wohnungen wie auch diejenige zur Schule oder zum Kindergarten,

h. die Stabilität, welche die Weiterführung der bisherigen Regelung mit sich bringt,

i. die Beziehung zu Geschwistern, Stief- und Halbgeschwistern,

j. die Einbettung des Kindes in sein weiteres soziales Umfeld sowie

k. der Wille des Kindes. ${ }^{6}$

Um die (alleinige oder alternierende) Obhut über sein 6 Kind auszuüben, muss ein Elternteil zwingend erziehungsfähig sein (Kriteriuma.). Die Relevanz der anderen Faktoren hängt von den konkreten Umständen des Einzelfalles ab. ${ }^{7}$ Bezüglich elterlicher Konflikte (Kriterium d.) präzisiert das Gericht:

"Allein aus dem Umstand, dass ein Elternteil sich einer alternierenden Betreuungsregelung widersetzt, kann indessen nichtohne Weiteres auf eine fehlende Kooperationsfähigkeit der Eltern geschlossen werden, die einer alternierenden Obhut im Wege steht. Ein derartiger Schluss könnte nur dort in Betrachtfallen, wo die Eltern aufgrund der zwischen ihnen bestehenden Feindseligkeiten auch hinsichtlich anderer Kinderbelange nicht zusammenarbeiten können, mit der Folge, dass sie ihr Kind im Szenario einer alternierenden Obhut dem gravierenden Elternkonflikt in einer Weise aussetzen würden, dieseinen Interessen offensichtlich zuwiderläuft.»8

4 Die alternierende Obhut ist eine Betreuungs- und Lebensform für Kinder getrenntlebender Eltern, bei welcher die Kinder zu ungefähr gleichen Teilen bei beiden Eltern leben (Urteil des Bundesgerichts 5A_46/2015 vom 26. Mai 2015 E. 4.4.3).

5 BGE142III612 E. 4.2; BGE142 III 617 E. 3.2.3.

6 Zum Ganzen: BGE142 III612 E. 4.3; BGE142 III 617 E. 3.2.3; Die für sinnvoll erachteten Kriterien b., e., und die Ergänzung zu g. entstammen dem Urteil des Bundesgerichts 5A_46/2015 vom 26. Mai 2015 E. 4.4.2 und 4.4.5.

7 BGE142 III612 E.4.3 und BGE142 III 617 E. 3.2.3 je m.w.H.

8 BGE142 III612 E.4.3. 
7 Sind die notwendigen Voraussetzungen für eine alternierende Obhut erfüllt, ist diese anzuordnen. ${ }^{9}$ Ist dies nicht der Fall, kommt es zu einem zweiten Schritt. Darin ist anhand derselben Kriterien zu ermitteln, in wessen Obhut es dem Kind voraussichtlich besser gehen wird.10 Dabei ist namentlich der Bereitschaft und Fähigkeit der Eltern Rechnung zu tragen, die Beziehung des Kindes zum anderen Elternteil zu fördern (Kriterium e.). ${ }^{11}$

\section{Urteil 5A_629/2019 vom 13. November 2020}

8 Nachfolgend werden die wichtigsten Aspekte des Sachverhalts und der Erwägungen in Bezug auf die alternierende Obhut beschrieben. Sämtliche nicht weiter spezifizierten Referenzen in Kapitel III. beziehen sich auf das Urteil des Bundesgerichts 5A_629/2019 vom 13. November 2020.

\section{Sachverhalt und Prozessgeschichte}

9 Im vorliegenden Entscheid ging es um die Betreuungsregelung dreier Kinder (C, D und E) der nicht verheirateten Eltern A und B. Während des Zusammenlebens betreute die Mutter B. die Kinder. Die Eltern trennten sich im August 2016 (z.G. Sv. A.a).

10 Bis zum Frühsommer 2017 führten die Eltern eine Mediation durch, die schliesslich scheiterte. In diesem Zeitraum betreuten die Eltern ihre Kinder während 5-8 Monaten in einer alternierenden Obhut (Sv. A.b; E. 8.4.1). Danach lebten die Kinder erneut mehrheitlich bei B. Letztere lebte inzwischen bei ihrem neuen Partner. Im Jahre 2018 bekamen die beiden einen Sohn (Sv. A.c).

11 Am 5.Juni 2017 verlangte der Vater A beim Zivilkreisgericht Basel-Landschaft Ost eine alternierende Obhut mit hälftiger Betreuungsaufteilung (Sv. B.a). Die Gerichtspräsidentin ordnete vorläufig jedoch nur ein ausgeweitetes Besuchsrecht an. Dagegen führte der Vater Berufung. Im Berufungsverfahren einigten sich die Eltern auf ein ausgeweitetes Besuchsrecht von Freitag um 19:30 Uhr bis Sonntag um 19:30 Uhr alle 14 Tage sowie wöchentlich von Donnerstag um 13:30 Uhr bis Freitag um 19:30 Uhr. Zu dessen Umsetzung wurden eine Erziehungsbeistandschaft und, auf Antrag beider Parteien, die Erstellung eines Gutachtens angeordnet (Sv. B.b f.).

\footnotetext{
9 BGE142 III 612 E.4.4; BGE142 III 617 E. 3.2.4; so auch Tagesanzeiger vom 14. März 2017 («Die Hoffnungen der Väter sind berechtigt»).

10 BGE142 III 612 E. 4.4; BGE142 III617 E. 3.2.4; Für nicht miteinander verheiratete Eltern gelten dieselben Kriterien (Urteil des Bundesgerichts 5A_707/2019 vom 18. August 2020 E.3.1.1).

11 BGE142III612 E. 4.4; BGE142III617 E.3.2.4.
}

Das Gutachten kam zum Ergebnis, die Eltern sollten die 12 Kinder hälftig betreuen (Sv. B.c). Dennoch teilte die Gerichtspräsidentin, am 8. November 2018, die Obhut der Mutter zu (Sv. B.d). Noch am selben Tag erhob A Berufung und hielt im Wesentlichen an seiner Forderung fest (Sv. C.a). Am 28. Mai 2019 wies das Kantonsgericht den Obhutsantrag ab und stellte A den Entscheid am 16.Juli 2019 zu (Sv. C.c).

A beantragte am 14. August 2019 beim Bundesgericht die 13 Aufhebung dieses Entscheids und die Gutheissung der alternierenden Obhut mit je hälftiger Betreuung (Sv. D.a). Das Bundesgericht trat auf die Beschwerde ein (E. 1), hob den Entscheid auf und wies ihn zur Ergänzung des Sachverhalts und Neubeurteilung an die Vorinstanz zurück (E.8.9).

\section{Präzisierung der allgemeinen Anforderungen}

Das Bundesgericht präzisiert zunächst zwei Kriterien sei- 14 ner Rechtsprechung: Zum Erfordernis, dass Eltern fähig und bereit sind, in Kinderbelangen zu kommunizieren und zu kooperieren, führt das Gericht aus, dass es unproblematisch ist, wenn die Eltern für die Kommunikation und Kooperation auf die Vermittlung durch eine Drittperson angewiesen sind (E. 4.2).

Bezüglich der Möglichkeit, das Kind persönlich zu betreu- 15 en, erwägt das Bundesgericht, dass dies vor allem dann eine Rolle spielt,

- wenn spezifische Bedürfnisse des Kindes eine persönliche Betreuung notwendig erscheinen lassen oder

- wenn ein Elternteil selbst in Randzeiten nicht bzw. kaum zur Verfügung steht.

Liegen keine derartigen Ausnahmen vor, ist von der 16 Gleichwertigkeit von Eigen- und Fremdbetreuung auszugehen (z.G. E. 4.2).

\section{Anforderungen an das Abweichen von Gutachten}

Das Bundesgericht hält fest, dass ein Gericht ein Gutach- 17 ten einholen darf, aber nicht muss. Liegt ein Gutachten vor, darf in Fachfragen nur aus triftigen Gründen davon abgewichen werden. Das Gericht hat dabei zu prüfen, ob ernsthafte Einwände gegen die Schlüssigkeit der gutachterlichen Darlegungen bestehen. Erscheint diese zweifelhaft, muss das Gericht ergänzende Beweise zur Klärung der Zweifel erheben. Das Abstellen auf eine nicht schlüssige Expertise oder der Verzicht auf gebotene zusätzliche Beweiserhebungen kann gegen das Verbot willkürlicher Beweiswürdigung verstossen (z.G. E. 7.1). 

zu ziehen sind, entscheidet allein das Gericht. Darum darf es grundsätzlich von der diesbezüglichen Empfehlung eines Gutachtens abweichen (z.G. E. 7.2).

\section{4. Überprüfung der Gründe für die Abkehr von der alternierenden Obhut}

19 Schliesslich prüft das Bundesgericht, ob die Vorinstanz zu Recht davon ausging, die Voraussetzungen für die alternierende Obhut seien nicht erfüllt, und analysiert mehrere Argumente der Vorinstanz (E. 8).

\section{a) Elterliche Kooperation und Kommunikation} an eine alternierende Obhut nicht, da diese nur mit Hilfe der Erziehungsbeiständin und eines Büchleins, in welches sie sich jeweils Nachrichten hineinschreiben, funktioniere. Wegen der Schulpflicht und der Distanz zwischen den elterlichen Wohnungen (20 Minuten mit dem Auto) sei eine bessere Kommunikation erforderlich (z.G. E. 8.3.1).

21 Nach dem Bundesgericht hätte die Vorinstanz berücksichtigen müssen, dass sich die Kommunikation und Kooperation mit der Erziehungsbeistandschaft gebessert hat und dass die Parteien auch bei einem ausgeweiteten Besuchsrecht gleichermassen kommunizieren und kooperieren müssen (E. 8.3.2). Am Gutachten bemängle die Vorinstanz nur, es gehe nicht auf die Bedeutung der Konflikte für das Kindeswohl bei einer alternierenden Obhut ein, mache dazu aber keine eigenen Feststellungen (E. 8.3.3). Unter diesen Voraussetzungen wären weitere Abklärungen notwendig gewesen (E. 8.3.4).

\section{b) Bisherige Betreuungsregelung}

22 Die Vorinstanz verneinte die alternierende Obhut auch aufgrund des Kriteriums der Stabilität, welche die bisherige Betreuungsregelung mit sich bringt (E. 8.4.1).

23 Traditionelle Rollen vor einer Trennung sprechen gemäss dem Bundesgericht eher gegen die alternierende Obhut. Dies relativiere sich jedoch dadurch, dass die Parteien bereits während mindestens fünf Monaten erfolgreich eine alternierende Obhut praktiziert haben. Diese Zeitspanne sei insbesondere für junge Kinder lange genug, um sich an die alternierende Obhut zu gewöhnen (z.G. E. 8.4.2).

\section{c) Geografische Distanz} gen der geografischen Distanz zwischen den beiden Wohnorten ab. Eine 20-minütige Autofahrt ist nach dem höchsten Gericht jedoch keine grundsätzlich unzumutbare
Belastung für die Kinder. Die Überlegung der Vorinstanz, die eine Stunde dauernde Fahrt mit öffentlichen Verkehrsmitteln sei nicht zumutbar, verdiene keine Beachtung, da der Transport mit dem Auto stattfinde. Die Kinder müssten auch den Schulweg nicht allein bewältigen können (z.G. E. 8.5).

\section{d) Würdigung des Gutachtens}

Die Vorinstanz erwog, das Gutachten sei aus der Sicht der 25 Elterninteressen erfolgt und nicht auf das Kindeswohl eingegangen. Deshalb sei nicht darauf abzustellen (z.G. E. 8.6).

Diese Kritik ist gemäss dem letztinstanzlichen Entscheid 26 zwar teilweise berechtigt. Allerdings könne der Erschöpfungszustand von B. Auswirkungen auf das Kindeswohl haben. Die gesundheitliche Verfassung eines Elternteils ist ein für die Obhutsfrage rechtserheblicher Umstand. Darum hätte festgestellt werden müssen, ob eine Entlastung der Mutter durch die alternierende Obhut den Kindern besser gedient hätte als die angefochtene Regelung. Dies umso mehr angesichts der Geburt des vierten Kindes. Da die Vorinstanz diese Abklärung unterliess, habe sie die Untersuchungsmaxime (Art. 296 Abs.1ZPO12) verletzt (z.G. E. 8.6).

\section{e) Berücksichtigung des Kindeswillens}

Aus dem Gutachten geht hervor, dass der damals 6-jäh- 27 rige Sohn die alternierende Obhut mit hälftigen Betreuungsanteilen gut fand. Dies sei ein rechtserheblicher Umstand, den die Vorinstanz hätte thematisieren müssen (z.G. E. 8.7).

\section{f) Trennung von Halb-Geschwistern}

Die Regelung, wonach Geschwister nicht getrennt wer- 28 den sollten, zielt darauf ab, zu vermeiden, dass für volle Geschwister unterschiedliche Obhutsregelungen gelten. Der Beziehung zu Halbgeschwistern sei zwar Rechnung zu tragen, es sei aber normal, wenn die Obhutsregelungen unterschiedlich sind. Eine Beziehung zu den Halbgeschwistern könne auch so gepflegt werden (z.G. E. 8.8).

\section{Begründung der Aufhebung des Entscheids}

Das Bundesgericht gelangt zum Schluss, dass die Vor- 29 instanz ihr Ermessen bundesrechtswidrig ausgeübt hat. Sie schloss, ohne eine Beeinträchtigung des Kindeswohls festzustellen, dass der Elternkonflikt die alternierende Obhut mit hälftiger Betreuung nicht zulasse. Zudem liess sie weitere rechtserhebliche Umstände ausser Acht (z.G. E. 8.9).

12 Schweizerische Zivilprozessordnung vom 19. Dezember 2008 (Zivilprozessordnung, ZPO; SR 272). 


\section{Urteil 5A_367/2020 vom 19. Oktober 2020}

30 Nachfolgend werden die wichtigsten Aspekte des Sachverhalts und der Erwägungen in Bezug auf die alternierende Obhut beschrieben. Sämtliche nicht weiter spezifizierten Referenzen in Kapitel IV. beziehen sich auf das Urteil des Bundesgerichts 5A_367/2020 vom 19. Oktober 2020.

\section{Sachverhalt und Prozessgeschichte}

31 A.A und B.A heirateten im Jahre 2009. C.A kam im Jahre 2016 zur Welt. Die Eltern teilten sich die Betreuungsaufgaben (Sv. A). Seit dem 13. April 2018 leben sie getrennt. Durch das Verschulden der Mutter B.A brach der Kontakt zwischen C.A und seinem Vater A.A bis im November 2018 ab (Sv. A; E. 3.4.1). Dennoch blieb deren Beziehung intakt (E.3.4.1).

Am 25. Oktober 2018 regelte das Bezirksgericht das Getrenntleben. Es teilte die Obhut über C.A der Mutter zu. A.A erhielt ein Besuchsrecht (Sv. B.a). Gegen den Entscheid erhoben beide Parteien Berufung ans Obergericht. Dieses ordnete neu ein ausgeweitetes Besuchsrecht an (z.G.Sv. B.b).

33 Gegen diesen Entscheid erhob der Vater Beschwerde ans Bundesgericht. Das Bundesgericht hob das Urteil des Obergerichts am 17. Oktober $2019^{13}$ auf und wies es zur Ergänzung des Sachverhalts und Neubeurteilung in der Sache an die Vorinstanz zurück (Sv. B.c). Diese ergänzte ihr Urteil am 6. April 2020 um eine Weisung an die Eltern: Sie sollten Hilfe für eine bessere und kindzentrierte Kommunikation suchen (Sv. C). A.A beschwerte sich beim Bundesgericht und ersuchte um eine alternierende Obhut (Sv.D.a).

undesgericht erachtet die Beschwerde für zulässig und die Begehren des A.A für hinreichend bestimmt (E.1.1f.). Es heisst die Beschwerde teilweise gut und weist die Sache an die Vorinstanz zurück, um die Modalitäten einer alternierenden Obhut zu regeln (z.G. E. 3.7 sowie Dispositiv Ziff. 1).

\section{Ausschliessliche Prüfung auf Willkür}

Beim vorliegenden Urteil handelt es sich um einen Entscheid im Rahmen eines Eheschutzverfahrens. Eheschutzentscheide sind Entscheide über vorsorgliche Massnahmeni.S.v. Art.98 BGG ${ }^{\mathbf{1 4}}$. Dies hat weitreichende Konsequenzen:

13 Es handelt sich um das Urteil des Bundesgerichts 5A_312/2019 vom 17. Oktober 2019.

14 Bundesgesetz über das Bundesgericht vom 17.Juni 2005 (Bundesgerichtsgesetz, BGG; SR 173.110).
- Es können nur Verletzungen verfassungsmässiger Rechte gerügt werden (Art. 98 BGG), alle anderen Beschwerdegründe (Art. 95 ff. BGG) sind ausgeschlossen, 15

- die Anwendung von Bundesgesetzen prüft das Bundesgericht nur auf die Verletzung des Willkürverbots (Art. 9 BV16) hin (z.G. E. 2.1). ${ }^{17}$

Dass das Bundesgericht seinen Entscheid ausschliesslich 36 unter Willkürgesichtspunkten fällte, macht das vorliegende Urteil besonders wichtig.

\section{3. Überprüfung der Gründe für die Abkehr von der alternierenden obhut}

Nach der Ergänzung des Sachverhalts sagte das Ober- 37 gericht, dass weder die Kommunikations- und Kooperationsfähigkeit der Eltern noch die Wohnsituation der Parteien ein Hindernis für die alternierende Obhut seien. Allerdings spreche die Stabilität der Verhältnisse bei Kleinkindern gegen eine alternierende Obhut (z.G. E. 3.2).

\section{a) Allgemein gehaltene Gründe}

Das Obergericht führte mehrfach sehr allgemein gehal- 38 tene Gründe auf, um die Abkehr von der alternierenden Obhut zu rechtfertigen. Es erwog:

- die alternierende Betreuung könne insbesondere für Säuglinge und Kleinkinder bis fünf Jahre zu einem Entwicklungsrisiko werden. Aus psychologischer Sicht werde angenommen, dass den besonderen Bindungs- und Betreuungsbedürfnissen von Säuglingen und Kleinkindern in der alternierenden Obhut kaum kindesgerecht Rechnung getragen werden könne (z.G. E. 3.3),

- ein Kind entwickle seine Bindung zu einer zweiten Bindungspersoni.d.R. erst mit 18 Monaten. Während des Zusammenlebens sei der Sohn der Parteien aufgrund des Alters nicht in der Lage gewesen, zu beiden Parteien eine gleich tragfähige Bindung aufzubauen. Darum sei davon auszugehen, dass die Mutter die erste Bindungsperson sei und zum Vater keine tragfähige Beziehung bestehe (z.G.E.3.5),

15 Auch Art.105BGG ist nicht anwendbar (MARKUS SCHOTT, in: Niggli/ Uebersax/Wiprächtiger/Kneubühler [Hrsg.], Basler Kommentar, Bundesgerichtsgesetz, 3. Aufl., Basel 2018, Art. 98 BGG N 8 [zit. BSK BGG-SCHOTT]).

16 Bundesverfassung der Schweizerischen Eidgenossenschaft vom 18. April 1999 (BV; SR101).

17 Siehe auch: Urteil des Bundesgerichts 5A_857/2016 vom 8. November 2017 E. 2. Über die bisherige Aufzählung hinaus gilt das qualifizierte Rügeprinzip: Die Verletzung verfassungsmässiger Rechte wird nur geprüft, wenn eine entsprechende Rüge gemacht wurde (Art.106 Abs. 2 BGG; BSK BGG-SCHOTT, Art. 98 N 8). Zudem prüft das Bundesgericht nur klar und detailliert erhobene und soweit möglich belegte Rügen (BSK BGG-SCHOTT, Art. 98 N18). Vgl. z.G. E. 2.1. 
- die ständigen Wechsel des Wohnorts seien wegen der Notwendigkeit, die Sachen immer wieder packen zu müssen, eine Belastung für das Kind(E. 3.6).

Mit Verweis auf seine bisherige Rechtsprechung18 erklärt das Bundesgericht, dass es von den konkreten Umständen abhängt, ob die alternierende Obhut angeordnet wird oder nicht (E. 3.3). Das blosse Aufwerfen der Frage nach der notwendigen Reife des Kindes, um Wechsel zu bewältigen (E. 3.6), reine Mutmassungen bezüglich der Qualität der Eltern-Kind-Beziehung (E. 3.5) oder das pauschale Abstellen auf kinderpsychologische Studien und Literatur (E. 3.3) genüge für einen willkürfreien Entscheid nicht (E. 3.5 mit Verweis auf E. 3.3). Gerade in der Literatur liessen sich Meinungen für jede Position finden (E. 3.3). ${ }^{19}$

\section{b) Stabilität der Verhältnisse}

40 Vor der Trennung betreuten die Eltern das Kind im Verhältnis von ca. 30:70\%. Der Kontaktabbruch zwischen C.A und A.A wurde durch B.A verursacht. Die Kind-Vater-Beziehung blieb aber bestehen. Dennoch sei wegen des Kriteriums der Stabilität keine alternierende Obhut angebracht. Dies auch wegen der «frühkindlichen Amnesie», der Betreuungs- und Umgebungsverhältnisse und des Säuglingsalters (z.G. E. 3.4.1).

41 Das Bundesgericht hält fest, dass die Stabilität ein Kriterium ist, das geprüft werden muss. Dabei muss gefragt werden, was die Weiterführung der bisherigen Regelung dem Kind bringt. Bei diesem Kriterium spricht der Umstand, dass vor der Trennung bereits eine alternierende Obhut gelebt wurde, für die alternierende Obhut. Indem die Vorinstanz im Wesentlichen nur auf die frühkindliche Amnesie abstelle und die Betreuungsaufteilung vor der Trennung völlig ignoriere, habe sie ihr Ermessen qualifiziert und fehlerhaft ausgeübt und sei in Willkür verfallen. Zudem könne das missbräuchliche Verhalten von B.A nicht dadurch gewürdigt werden, indem die alleinige Obhut mit dem Kontaktabbruch begründet werde (z.G. E.3.4.3).

\section{Begründung der Aufhebung des Entscheids}

undesgericht erachtet den Ermessensentscheid im Ergebnis für willkürlich und stellt fest, dass ohne sachlich haltbare Gründe von der konstanten bundesgerichtlichen Rechtsprechung abgewichen wurde. Die Voraussetzungen für eine alternierende Obhut seien erfüllt (z.G. E.3.7).

\section{BGE142 III 402 E. 4.2.}

19 Für eine von der Ansicht des Obergerichts abweichende Auffassung siehe z.B.: RICHARD A. WARSHAK, Social Science and Parenting Plans for Young Children: A Consensus Report, Psychology, Public Policy, and Law 2014, S. 46 ff.

\section{Bedeutung für die Praxis}

Mit den beiden Urteilen hat das Bundesgericht seine 43 Rechtsprechung bestätigt und präzisiert. Hier werden ihre Folgen für die Praxis erläutert.

\section{Alleinige Obhut nur bei konkreten Gründen}

Sind sich Eltern über die Betreuungsregelung uneinig, 44 verlangt das Bundesgericht in einem ersten Schritt, dass «gestützt auf festgestellte Tatsachen der Gegenwart und der Vergangenheit eine sachverhaltsbasierte Prognose darüber» erstellt wird, ob die alternierende Obhut mit dem Kindeswohl vereinbar ist oder nicht. ${ }^{20}$ Dazu ist anhand mehrerer Kriterien ${ }^{21}$ zu prüfen, ob im Einzelfall konkrete Gründe gegen die alternierende Obhut sprechen. ${ }^{22}$ Ist dies der Fall, ist die alleinige Obhut anzuordnen, wenn dies zu einem für das Kind günstigeren $\mathrm{Er}$ gebnis führt. Abweichungen von dieser Rechtsprechung können gegen das Willkürverbot verstossen. ${ }^{23}$

Mit den Urteilen verdeutlicht das Bundesgericht, dass 45 es nicht gewillt ist, die Verneinung der alternierenden Obhut mit pauschal gehaltenen Gründen zu billigen. Es genügt nicht, die alternierende Obhut mit allgemeinen Argumenten zu verneinen, wie z.B. die alternierende Obhut sei schlecht für junge Kinder. ${ }^{24}$

\section{Alternierende Obhut bei fehlender günstigerer Alternative}

In einem der Fälle sah die Vorinstanz wegen Kommuni- 46 kationsschwierigkeiten von der alternierenden Obhut ab und ordnete eine alleinige Obhut mit ausgeweitetem Besuchsrecht an. ${ }^{25}$ Dass die Kommunikationsschwierigkeiten für diese Betreuungsregelung ebenso problematisch waren wie für die alternierende Obhut, liess sie ausser Acht. ${ }^{26}$

Es sind weitere Fälle denkbar, in welchen konkrete Grün- 47 de sowohl gegen die alternierende als auch gegen die alleinige Obhut und ihre Varianten sprechen. Würde eine alternierende Obhut aus solchen Gründen abgelehnt,

20 Urteil des Bundesgerichts 5A_629/2019 vom 13. November 2020 E. 4.1; BGE142 III 612 E. 4.2.

21 Siehe II.

22 Urteil des Bundesgerichts 5A_367/2020 vom 19. Oktober 2020 E.3.3.

23 Urteil des Bundesgerichts 5A_367/2020 vom 19. Oktober 2020 E. 3.7; Vgl. auch Urteil des Bundesgerichts 5A_629/2019 vom 13. November 2020 E.8.9.

24 Urteil des Bundesgerichts 5A_367/2020 vom 19. Oktober 2020 E.3.3 i.V.m. E.3.7.

25 Urteil des Bundesgerichts 5A_629/2019 vom 13. November 2020 E. 8.3.1.

26 Urteil des Bundesgerichts 5A_629/2019 vom 13. November 2020 E. 8.3.2. 
obwohl die angeordnete Alternative zu schlechteren Ergebnissen für das Kind führt, wäre dem Kindeswohl wenig gedient. Es macht darum wenig Sinn, von einer alternierenden Obhut abzusehen, wenn zwar konkrete Gründe gegen sie sprechen, aber keine für das Kind günstigere Alternative besteht.

\section{Priorisierung höherer Betreuungsanteile}

Das Bundesgericht dürfte die alternierende Obhut mit hälftigen Betreuungsanteilen derjenigen mit ungleichen Betreuungsanteilen oder dem ausgeweiteten Besuchsrecht tendenziell vorziehen. ${ }^{27}$ Allerdings hat das Bundesgericht diesen Vorzug bisher nur bei Betreuungsanteilen in der Höhe des besprochenen Urteils gewährt. Die Betreuungsanteile des Vaters beliefen sich darin auf fünf Tage und sechs Stunden alle 14 Tage. ${ }^{28}$ Hälftige Betreuungsanteile tendenziell auch gegenüber gleicheren Anteilen als diesen zu priorisieren würde zwar Sinn machen, da es zu einer grösseren Rechtssicherheit und einer Reduktion der Konflikte führen dürfte. ${ }^{29}$ Das Gericht hat die Frage aber noch nicht geklärt.

\section{Unterstützungsbedürftige Kommunikation und Kooperation genügt}

49 Es genügt für die alternierende Obhut, wenn die Eltern für eine ausreichende Kooperation und Kommunikation auf die Vermittlung einer Drittperson oder Hilfsmittel, wie etwa ein Büchlein, angewiesen sind. Sie müssen nicht zwingend allein kommunizieren und kooperieren können. ${ }^{30}$

\section{Gleichwertigkeit der Fremdbetreuung}

50 Das Bundesgericht präzisiert, dass Eigen- und Fremdbetreuung grundsätzlich gleichwertig sind. Von diesem Grundsatz ist nur unter speziellen Umständen (spezifische gesundheitliche Bedürfnisse eines Kindes oder [fast] gänzlicheUnverfügbarkeit eines Elternteils) abzusehen. ${ }^{31}$ Ein Elternteil dürfte folglich i.d.R. keine Nachteile mehr in Kauf nehmen müssen, weil er erwerbstätig ist und die Kinder darum fremdbetreuen lassen muss.

27 Vgl. Urteil des Bundesgerichts 5A_629/2019 vom 13. November 2020 Sv. B.b, B.d und C.c sowie E. 8.3.2, 8.3.4 und 8.9.

28 Vgl.Urteil des Bundesgerichts 5A_629/2019 vom 13. November 2020 Sv. B.b, B.d und C.c.

29 Ausführlich dazu z.B.: SANFORD BRAVER, The costs and pitfalls of individualizing decisions and incentivizing conflict: A comment on AFCC's think tank report on shared parenting, Family Court Review 2014, S. $175 \mathrm{ff}$.

30 Z.G. Urteil des Bundesgerichts 5A_367/2020 vom 19. Oktober 2020 E. 4.2, 8.3.1ff. und 8.9.

31 Urteil des Bundesgerichts 5A_629/2019 vom 13. November 2020 E. 4.2; Siehe auch: Urteil des Bundesgerichts 5A 707/2019 vom 18. August 2020 E. 3.1.1 sowie ferner: BGE144 III 481 E. 4.6.3 ff. m.w.H.

\section{Geografische Distanz}

Eine 20-minütige Autofahrt ist für Kinder grundsätzlich 51 zumutbar. Ausserdem müssen Kinder den Schulweg nicht allein bewältigen können. Rein hypothetische Aspekte, wie die Zeit, die benötigt wird, um mit öffentlichen Verkehrsmitteln von einer Wohnung zur anderen zu gelangen, sind nicht zu berücksichtigen, wenn der Weg ohnehin mit dem Auto zurückgelegt wird. ${ }^{32}$

\section{Berücksichtigung des Kindeswillens}

Das Bundesgericht schreibt vor, dass der Kindeswille in 52 allen Entscheiden, die Kinder betreffen, berücksichtigt werden muss. ${ }^{33}$ Folglich muss in den für Kinder besonders relevanten Betreuungsentscheiden ersichtlich sein, dass die Wünsche der Kinder thematisiert wurden (vgl. auch Art.12 KRK ${ }^{34}$ ).

\section{Trennung von Halbgeschwistern}

Der Grundsatz, dass Geschwister nicht voneinander ge- 53 trennt werden, findet auf Halbgeschwister keine Anwendung. ${ }^{35}$ Von der alternierenden Obhut kann folglich nicht allein darum abgesehen werden, weil aus mehreren Partnerschaften Kinder hervorgegangen sind und Kinder aus der einen Partnerschaft nie von Kindern aus einer anderen Partnerschaft getrennt werden dürften. Gemäss der bisherigen Rechtsprechung kann es bei gegebenen Umständen genügen, dass Halbgeschwister Ihre Beziehung im Rahmen eines gewöhnlichen Besuchsrechts aufbauen oder pflegen. ${ }^{36}$ Zuweilen kann es auch Sinn machen, Vollgeschwister voneinander zu trennen. ${ }^{37}$

\section{Anforderungen an die Abweichung von Gutachten}

Grundsätzlich steht es einem Gericht frei, ein Gutachten 54 anzuordnen. Existiert ein Gutachten, kann in Fachfragen nur aus triftigen Gründen davon abgewichen werden. Dabei ist zu prüfen, ob ernsthafte Einwände bezüglich dessen Schlüssigkeit bestehen. Bleiben Zweifel, muss das Gericht ergänzende Beweise erheben und die offenen

32 Z.G. Urteil des Bundesgerichts 5A 629/2019 vom 13. November 2020 E. 8.5.

33 Urteil des Bundesgerichts 5A 629/2019 vom 13. November 2020 E. 8.7.

34 Übereinkommen über die Rechte des Kindes vom 20. November 1989 (Kinderrechtskonvention, KRK; SR 0.107).

35 Urteil des Bundesgerichts 5A_629/2019 vom 13. November 2020 E.8.8.

36 Urteil des Bundesgerichts 5A_844/2019 vom 17. September 2020 E. $3.4 \mathrm{f}$.

37 So z.B., wenn sich das Alter, die Bedürfnisse, die emotionalen Bindungen oder Wünsche der Kinder unterscheiden (Urteil des Bundesgerichts 5A_707/2019 vom 18. August 2020 E.3.1.1 m.w.H.). 
Fragen klären. In Rechtsfragen entscheidet das Gericht nach eigenem Ermessen. ${ }^{38}$

\section{Berichtigung zur «Regelfall-Diskussion»}

Im Zusammenhang mit diesem Urteil ist vermehrt der Begriff «alternierende Obhut als Regelfall» gefallen. ${ }^{39}$ In der Schweiz besteht grosse Unklarheit bezüglich dieses Begriffs. So hiess es z.B., dass wer dies fordere, alle Eltern zu einer alternierenden Obhut zwingen wollte-sogar dann, wenn sie sich auf eine alleinige Obhut geeinigt haben. ${ }^{40}$ Wie jemand zu einem solchen Bild gelangen konnte, ist eine offene Frage. Zumindest ist es wichtig klarzustellen, dass dies dem widerspricht, was in der Forschung unter einer alternierenden Obhut als Regelfall verstanden wird. Von selbst versteht sich, dass diese Lösung grundsätzlich nur dann Sinn macht, wenn sich die Eltern bezüglich der Obhut uneinig sind.

Fachpersonen verstehen unter der alternierenden $\mathrm{Ob}$ hut als Regelfall eine widerlegbare Vermutung zugunsten deralternierenden Obhut. ${ }^{41}$ Dies bedeutet, dass die alternierende Obhut gegenüber der alleinigen Obhut vorgezogen wird, wenn im Einzelfall keine konkreten Gründe dagegensprechen. So gesehen ist bereits der kleinste Vorzug der alternierenden Obhut ein «Regelfall». Eine Vermutung fördert die alternierende Obhut, muss aber nicht dazu führen, dass sie in der Praxis die meistverbreitete Betreuungsregelung wird.42 Je nach Höhe der Anforderungen, um die Vermutung zu widerlegen, liesse sich zwischen starken oder schwachen Vermutungen unterscheiden. ${ }^{43}$

38 Z.G.Urteil des Bundesgerichts 5A_629/2019 vom 13. November 2020 E.7.1f. und 8.3.3f.; vgl. auch BGE138III193 E.4.3.1.

39 CLAUDIA BLUMER, Bundesgericht macht alternierende Obhut zur Regel, Tagesanzeiger vom 10. Dezember 2020.

40 So hiess es z.B.: «In der Vernehmlassung ist gefordert worden, den Grundsatz der alternierenden Obhut im Gesetz zu verankern. (...) Aus den genannten Gründen erachtet es der Bundesrat nicht als angezeigt, alle getrennt lebenden Eltern zu einer alternierenden Obhut zu verpflichten» (Botschaft vom 29. November 2013 zu einer Änderung des Schweizerischen Zivilgesetzbuches [Kindesunterhalt; BBl 2014 529], S. 564f.). Oder es wurde suggeriert, bei einer «Regelfall-Lösung» würde die alternierende Obhut «in allen Fällen» angeordnet (Eidgenössisches Justiz- und Polizeidepartement [EJPD], Alternierende Obhut: In vielen Fällen sinnvoll und gut für das Kind, aber nicht in allen, 8. Dezember 2017).

41 Vgl. z.B.: SANFORD L. BRAVER / MICHAEL E. LAMB, Shared Parenting After Parental Separation: The Views of 12 Experts, Journal of Divorce \& Remarriage 2018, S. 380 .

42 Ausführlich dazu am Beispiel Spaniens: DIEGO BECERRIL RUÍZ / JOSÉ MANUEL JIMÉNEZ-CABELLO, Legislation and Family, Divorce and granting of custody, in: de Torres Perea / Kruk / Ortiz-Tallo (Hrsg.), The Routledge International Handbook of Shared Parenting and the Best Interest of the Child, Oxon 2021, S. $157 \mathrm{ff}$.

43 In diesem Sinne wurde z.B. in der Lehre diskutiert, wie hoch die Eingriffsschwelle für den Entzug der elterlichen Sorge (bzw. wie stark die Vermutung z.G. der gemeinsamen elterlichen Sorge) sein
Diese Vermutung wird in der Forschung ernsthaft dis- 57 kutiert und von zahlreichen Fachpersonen gefordert. Dies kam z.B. im Jahre 2014 sehr eindrücklich zur Geltung, als 111 Expertinnen und Experten der Psychologie in einem (sehr seltenen) Konsensbericht die alternierende Obhut für Kinder jeden Alters (also auch für Kleinkinder) zum Regelfall empfahlen. ${ }^{44}$ In einer Spezialausgabe zur alternierenden Obhut des Journal of Divorce \& Remarriage sprachen sich 12 Expertinnen und Experten mehrheitlich für eine Vermutung aus, da selbst bei elterlichen Konflikten fast alle empirischen Studien ergeben, dass Kinder von der alternierenden Obhut profitieren. 45 Auch in der Schweiz wurde, basierend auf grundrechtlichen Überlegungen, die Ansicht vertreten, die alternierende Obhut müsse der Ausgangspunkt in Betreuungsfragen sein. ${ }^{46}$ Schliesslich ist die «Regelfalllösung» bereits in mehreren Ländern etabliert. ${ }^{47}$ Die Ergebnisse einer systematischen Evaluation in Arizona waren sogar mehrheitlich positiv. ${ }^{48}$

Die alternierende Obhut als Regelfall muss auch im Lichte 58 der möglichen Alternativen betrachtet werden. Dies sind z.B. das Mutterprimat, die alleinige Obhut als Regelfall oder der Kindeswohlstandard («the child's best interests principle»), bei dem in jedem Einzelfall versucht wird, die beste Lösung für das Kind zu ermitteln und anzuordnen.

Letztere Lösung wirkt sympathisch, wird in der Lehre 59 aber stark kritisiert. Sogar Münzen werfen sei besser. 49 Angesehenste Fachpersonen heben zwei Probleme besonders hervor:

1. Die faktische Unmöglichkeit, das künftige Kindeswohl in jedem Fall zu ermitteln: Es ist unmöglich, in jedem Fall festzustellen, welches die in der Zukunft

SOll (WILHELM FELDER / HEINZ HAUSHEER / REGINA E. AEBI-MÜLLER/ ERICA DESCH, Gemeinsame elterliche Sorge und Kindeswohl, ZBJV 2014, S. $892 \mathrm{ff}$ )

44 WARSHAK (Fn. 19), S. 59 (Konsenspunkt1).

45 BRAVER/LAMB (Fn. 41), S.381f. mit Verweis auf: LINDA NIELSEN, Re-examining the research on parental conflict, coparenting, and custody arrangements, Psychology, Public Policy, and Law 2017, S. $211 \mathrm{ff}$.

46 HILDEGUND SÜNDERHAUF / MARTIN WIDRIG, Gemeinsame elterliche Sorge und alternierende Obhut, Eine ent wicklungspsychologische und grundrechtliche Würdigung, AJP 2014, S.903; MARTIN WIDRIG, Alternierende Obhut, Leitprinzip des Unterhaltsrechts aus grundrechtlicher Sicht, AJP 2013, S. 905 ff.; Zustimmend: STEPHAN BERNARD / BEDA MEYER LÖHRER, Kontakte des Kindes zu getrennt lebenden Eltern - Skizze eines familienrechtlichen Paradigmenwechsels, Jusletter vom 12. Mai 2014, Fn. 29.

47 So z.B.in Belgien, mehreren autonomem Gemeinschaften Spaniens oder in den USA (z.B. Arizona).

48 WILLIAM V. FABRICIUS / MICHAEL AARON / FAREN R. AKINS / JOHN J. ASSINI / TRACY MCELROY, Parenting Time? An evaluation of Arizona's new child custody statute, in: de Torres Perea / Kruk / Ortiz-Tallo (Hrsg.), The Routledge International Handbook of Shared Parenting and the Best Interest of the Child, Oxon 2021, S. $370 \mathrm{ff}$.

49 Vgl. z.B. ROBERT H. MNOOKIN, Child-Custody Adjudication: Judicial Functions in the Face of Indeterminacy, Law and Contemporary Problems 1975, S. $289 \mathrm{ff}$. 
beste Lösung für das Kind sein wird. Es wäre notwendig, immer alle möglichen Varianten, alle möglichen Ergebnisse dieser Varianten, die Wahrscheinlichkeiten, mit welcher diese Ergebnisse eintreten, und der Wert, der jedem dieser Ergebnisse beigemessen wird, zu ermitteln bzw. zu berücksichtigen. ${ }^{50}$ Dies ist faktisch unmöglich. ${ }^{51}$ Zudem kosten derart ambitionierte Entscheide wertvolle Zeit ${ }^{52}$ und viel Geld, das später den Kindern fehlt. ${ }^{53}$ Entscheide, die auf empirisch gestützten Grundsätzen aufbauen, führen zu besseren Ergebnissen. ${ }^{54}$

2. Probleme aufgrund des (zu) weiten Ermessens mit dem Kindeswohlbegriff: Wenn jede Instanz «frei wählen» darf, was sie als Kindeswohl bezeichnet, ist der Entscheid im Rechtsmittelverfahren nicht überprüfbar. ${ }^{55}$ Entscheide, die auf derart weitem Ermessen beruhen, basieren zudem häufig mehr auf persönlichen Überzeugungen als logisch nachvollziehbaren Gründen. ${ }^{56}$ Die Konsequenzen sind weniger Rechtssicherheit, schlechtere Kooperation und mehr Streit zwischen den Eltern. ${ }^{57}$

60 Da auch die psychologische Forschung in überzeugender Weise darlegt, dass übliche Besuchsrechte nicht im Interesse der Kinder sind und mehr Zeit mit beiden Eltern die Regel (und nicht die Ausnahme) sein sollte, ${ }^{58}$

50 JON ELSTER, Solomonic Judgements: Against the Best Interests of the Child, The University of Chicago Law Review 1987, S. $12 \mathrm{ff}$.; MNOOKIN, Child-Custody Adjudication (Fn. 49), S. $256 \mathrm{ff}$.

51 ROBERT H. MNOOKIN, Child Custody Revisited, Law and Contemporary Problems 2014, S. $251 \mathrm{ff}$; BRAVER (Fn. 29), S. $176 \mathrm{ff}$; ROBERT E. EMERY / ELIZABETH S. SCOTT, Gender Politics and Child Custody: The Puzzling Persistence of the Best-Interests Standard, Law and Contemporary Problems 2014, S. 92.

52 BRAVER (Fn. 29), S. 177; MICHAEL E. LAMB, Dangers associated with the avoidance of evidence-based practice, Family Court Review 2014 S. 195 .

53 BRAVER (Fn. 29), S. 177.

54 LAMB (Fn. 52), S. 195 f.; BRAVER (Fn. 29), S. 177; EMERY/SCOTT (Fn. 51) S.76.

55 GERARD BRENNAN in Judgement of the High Court of Australia, Secretary, Department of Health and Community Services v JWB and SMB (Re Marion) (1992) FLC 92-293 of 6 May 1992, N14.

56 GERARD BRENNAN in Judgement of the High Court of Australia, Se cretary, Department of Health and Community Services v JWB and SMB (Re Marion) (1992) FLC 92-293 of 6 May 1992, N14; MNOOKIN, Child Custody Revisited (Fn. 51), S. 252.

57 MNOOKIN, Child Custody Revisited (Fn. 51), S. $251 \mathrm{ff}$.; BRAVER (Fn. 29), S.176f.; EMERY/SCOTT (Fn. 51), S. 69.

58 JOAN B. KELLY, Paternal Involvement and Child and Adolescent Ad justment after Separation and Divorce: Current Research and Implications for Policy and Practice, International Family Law, Policy and Practice 2014/1, S. 17 f.; Für die wahrscheinlich umfassendste Übersicht aller Studienergebnisse siehe: LINDA NIELSEN, Joint versus sole physical custody: Children's outcomes independent of parent-child relationships, income, and conflict in 60 studies, Journal of Divorce \& Remarriage 2018, S. 247 ff. Zurückhaltender, allerdings ohne Berücksichtigung des Grossteils der Ergebnisse der quantitativen Forschung: MICHELLE COTTIER / ERIC D. WIDMER / SANDRINE TORNARE / MYRIAM GIRARDIN, Interdisziplinäre Studie zur alternierenden Obhut, Genf 2017, S. 28 ff. In der soweit ersichtlich einzigen
Trennungskinder seit Jahrzehnten mehr Zeit mit beiden Elternteilen wünschen ${ }^{59}$ und der alternierenden Obhut sehr gute Noten erteilen, ${ }^{60}$ scheint eine widerlegbare Vermutung zugunsten der alternierenden Obhut zumindest eine viable, dem Kindeswohl potenziell dienliche Lösung zu sein.

\section{Der Wille des Gesetzgebers}

Eine Lehrmeinung warf dem Bundesgericht vor, es ver- 61 stosse gegen den Willen des Gesetzgebers. Dieser habe die alternierende Obhut nur gewollt, wenn sie die beste Lösung sei. ${ }^{61}$

Diese Äusserungen könnten suggerieren, die alleinige 62 Obhut sei als Regel, die alternierende als Ausnahme gedacht. Dies ist schon darum unzutreffend, da der Bundesrat ursprünglich gewollt hatte, dass immer die im Einzelfall günstigste Lösung für das Kind angeordnet wird (Kindeswohlstandard). ${ }^{62}$ Der Gesetzgeber wich jedoch von diesem Vorschlag ab. Auf Initiative der Kommission für Rechtsfragen des Ständerats (RK-S) nahm er vier neue Bestimmungen zur alternierenden Obhut ins $\mathrm{ZGB}^{63}$ auf (Art. 298 Abs. ${ }^{\text {bis }}$ f. sowie Art. 298b Abs. $3^{\text {bis }}$ f. ZGB). Diese zielte damit auf ein grundsätzliches Recht des Kindes auf Betreuung durch beide Eltern ${ }^{64}$ und - stets unter Vorbehalt der Wahrung des Kindeswohls - einen besseren Schutz des Rechts aufBetreuung ab. ${ }^{65}$ Selbst die vom Bundesrat zur Erfüllung des Postulats 15.3003 in Auftrag gegebene interdisziplinäre Studie kommt zum Schluss: «Mit diesen Bestimmungen signalisiert der Gesetzgeber, dass er dieser egalitären Organisationsweise der gemeinsamen Elternschaft nach einer Trennung oder Scheidung den Vorzug gibt.» ${ }^{66}$ Auch die mehrheitlich

schweizerischen Studie mit einem erwähnenswerten Ergebnis zur alternierenden Obhut waren Eltern, welche die alternierende Obhut freiwillig vereinbarten, mit dieser Betreuungslösung mehrheitlich sehr zufrieden (ANDREA BÜCHLER / LINUS CANTIENI / HEIDI SIMONI, Die Regelung der elterlichen Sorge nach Scheidung de lege ferenda - ein Vorschlag, Fampra.ch 2007, S. 210).

59 KELLY (Fn. 58), S.17f.

60 Statt vieler: WILLIAM V. FABRICIUS, Listening to children of divorce: New findings that diverge from Wallerstein, Lewis, and Blakeslee, Family Relations 2003, S. 387 (ca. 70\% aller Studierenden und über 90\% derjenigen, die eine alternierende Obhut gelebt hatten, erachteten diese für die günstigste aller Betreuungslösungen).

61 ROLAND FANKHAUSER, zit. nach: BLUMER (Fn.39).

62 Botschaft zu einer Änderung des Schweizerischen Zivilgesetzbuches (Kindesunterhalt) (Fn. 40), S. 565 f.; So auch: SÜNDERHAUF/ WIDRIG (Fn.46), S. 903 m.W.H.

63 Schweizerisches Zivilgesetzbuch vom 10. Dezember 1907 (ZGB; SR 210).

64 Votum Ständerat Stadler, AB 2014 S1120; Votum Ständerat Janiak, AB 2014 S1121 sowie 1122.

65 Ausführlich zu den Hintergründen, die zu den Bestimmungen geführt haben siehe Votum Ständerat Janiak, AB 2014 S1121.

66 COTTIER et al. (Fn. 58), S. 18 . 
erst skeptische Kommission für Rechtsfragen des Nationalrats (RK-N) hielt im ersten Satz dieses Postulats fest: «Die alternierende Obhut muss gefördert werden.» ${ }^{67}$

Der Gesetzgeber hat kein bestimmtes Betreuungsmodell vorgeschrieben. ${ }^{68}$ Er sagte auch, dass die alternierende Obhut keine exakt hälftige Betreuungsaufteilung implizieren muss. ${ }^{69}$ Die genaue Ausgestaltung des Betreuungsrechts überliess er, genau wie diejenige des Unterhalts- und Sorgerechts, der Praxis und damit in erster Linie dem Bundesgericht. Dies bedeutet jedoch nicht, dass Gerichte und Behörden völlig frei wären zu machen, was sie wollen. Der Gesetzgeber hat deutlich mitgeteilt, was er erwartet:

- Das Betreuungsrecht ist im Lichte der Sorgerechtsrevision zu betrachten. ${ }^{70}$ Diese stand im Zeichen des Kindeswohls und (nachgeordnet) der Gleichstellung. ${ }^{71}$

- Die frühere Praxis, wonach für die Betreuungslösung nach der elterlichen Trennung einfach auf die bisherige Rollenteilung abgestellt wird, ist aufzugeben. Sie relativiert bei ernsthafter Bereitschaft, das Kind zu betreuen, das Prinzip der gemeinsamen elterlichen Sorge. ${ }^{72}$

- Die alternierende Obhut soll namentlich auch dann möglich sein, wenn vor der Trennung eine traditionelle Rollenaufteilung herrschte. ${ }^{73}$ Sollte die alternierende Obhut zu einer Reduktion des Unterhaltsanspruchs führen, sei dies hinzunehmen. ${ }^{74}$ Die alleinige Obhut nur dazu anzuordnen, um mehr Unterhalt zuzusprechen, dürfte darum unzulässig sein. ${ }^{75}$

Bundesrätin Sommaruga hob noch etwas hervor: Die Überzeugung des Gesetzgebers, dass eine bedeutsame Beziehung zu beiden Eltern im Interesse des Kindes ist. ${ }^{76}$ Diese aufrecht zu erhalten sei bei einer «Sonntagsbeziehung» schwierig. 77 «Regelmässige persönliche Beziehun-

67 Postulat 15.3003, Alternierende Obhut. Klärung der Rechtsgrundlagen und Lösungsvorschläge vom 23. Januar 2015 der RK-N.

68 Votum Ständerat Stadler, AB 2015S188.

69 Votum Ständerat Stadler, AB 2015S188.

70 Votum Ständerat Stadler, AB 2014 S1120; Votum Bundesrätin Sommaruga, AB2014S1122.

71 Statt vieler: Votum Ständerätin Savary, AB 2014 S 1120. Vgl. auch: Votum Nationalrat Wehrli, AB 2005N1495.

72 Votum Ständerat Janiak, AB 2014 S1121.

73 Votum Ständerat Stadler, AB 2014 S1120; Votum Ständerat Cramer, AB2015S189.

74 Votum Ständerat Cramer, AB 2015S189.

75 Für Wege, um Unterhalt zuzusprechen, siehe Votum Nationalrätin Schneider Schüttel, AB 2015 N 423; Votum Ständerat Stadler, AB 2014 S1120; Votum Ständerat Janiak, AB 2014 S 1121. Eine Möglichkeit wäre auch die Einführung eines «kompensatorischen Unterhalts», bei welchem ein Elternteil für finanzielle Nachteile einer vereinbarten Betreuungsregelung entschädigt würde.

76 AB2015S189; siehe auch Votum Ständerat Engler, AB 2015 S187.

77 AB2015S189. gen zu beiden Elternteilen kann man dann am besten sicherstellen, wenn eine alternierende Obhut vorgesehen wird.» 78

Global betrachtet sprechen die vom Gesetzgeber festge- 65 haltenen Grundsätze und Erwartungen deutlich gegen eine alleinige Obhut als Regelfall und zugleich für einen tendenziellen Vorzug der alternierenden Obhut.

\section{Würdigung}

Das Bundesgericht macht die alternierende Obhut zum 66 Ausgangspunkt in Betreuungsfragen, wenn sich Eltern uneinig sind. Von der Idee eines grundsätzlichen Rechts auf Alleinbetreuung und Unterhalt hat es damit, zu Recht, Abstand genommen. An der bundesgerichtlichen Methode besonders positiv zu werten ist, dass die wichtigsten Risikofaktoren für das Kindeswohl im Einzelfall geprüft werden müssen. Diese Vorsichtsmassnahme erhöht die Wahrscheinlichkeit, dass allfällige Risiken für das Kind entdeckt werden, und gewährleistet damit einen besonders guten Kindesschutz. Diese Rechtsprechung verkörpert ferner beide Charakteristiken einer guten Rechtslage: Sie schafft zum einen ein Leitbild, das auch in unproblematischen Fällen daran erinnert, dass eine gemeinsame Betreuung und eine bedeutsame Beziehung zu beiden Eltern im Interesse des Kindes sind. ${ }^{79}$ In Schweden hat u.a. genau dieses Bewusstsein der Eltern zur Verbreitung der alternierenden Obhut beigetragen. ${ }^{80}$ Zum andern bietet die Rechtsprechung auch die notwendigen Werkzeuge für den Umgang mit schwierigen Fällen.

Die Urteile dürften, nebst dem Dienst für das Kind, auch 67 elterliche Konflikte reduzieren, Rechtssicherheit und die richtigen Anreize schaffen: Die alternierende Obhut, die Gleichbehandlung von Fremd- und Eigenbetreuung sowie neuere Entwicklungen im Unterhaltsrecht ${ }^{81}$ werden die Erwerbstätig- und Selbständigkeit beider Eltern fördern, was wiederum das Armutsrisiko der Kinder massiv reduzieren wird. ${ }^{82}$ Dies sind wichtige, positive

\section{Votum Bundesrätin Sommaruga, AB 2015N 424.}

\section{Vgl. Votum Ständerat Engler, AB2015S187.}

80 EMMA FRANSSON / ANNA SARKADI / ANDERS HJERN / MALIN BERGS TRÖM, Why should they live more with one of us when they are children to us both? Parents' motives for practicing equal joint physical custody for children aged 0-4, Children and Youth Services Review 2016, S. 159.

81 Pressemitteilung des Bundesgerichts vom 9. März 2021 (Urteile [5A_907/2018,5A_311/2019, 5A_891/2018,5A_104/2018, 5A_800/2019], Einheitliche Berechnungsmethode beim familienrechtlichen Unterhalt).

82 Organisation for Economic Co-operation and Development (OECD), Doing Better for Families, 2011, Table1.3, S. 41. Aus der Tabelle wird ersichtlich, dass sich das Armutsrisiko von Kindern in Alleinerziehendenhaushalten um den Faktor drei und in «intakten» Familien um den Faktor vier reduziert, wenn beide Eltern erwerbstätig sind. 
Neuerungen im schweizerischen Recht. Davon profitieren Kinder, Eltern und die ganze Gesellschaft.

\section{Fazit}

Das Bundesgericht macht die alternierende Obhut zum Ausgangspunkt in Betreuungsfragen. Von ihr darf nur abgewichen werden, wenn konkrete Gründe gegen sie und für eine andere Betreuungslösung sprechen. Zugleich hält es den Kindesschutz besonders hoch, indem es in jedem Fall eine Prüfung der Risikofaktoren für das Kind verlangt.

Diese vorsichtige Förderung der alternierenden Obhut 69 entspricht dem Willen des Gesetzgebers und wird durch die Ergebnisse der empirischen Forschung gestützt. Mit seiner Rechtsprechung schafft das Bundesgericht somit nicht nur Rechtssicherheit, sondern höchstwahrscheinlich auch die bestmögliche Lösung für das Kind. 